DOI: $10.32481 / \mathrm{djph} .2021 .07 .018$

\title{
From the History and Archives Collection
}

Sharon Folkenroth-Hess, M.A.

Collections Manager, Delaware Academy of Medicine / Delaware Public Health Association

"Healthcare, more than any other profession, undergoes constant change. The rapid progress of science necessitates a continuous revision of the methods and techniques to maintain or restore human health. Practitioners of medicine must stay informed of new developments to offer the best possible care to patients." Preamble to the Resolution to Form the Delaware Academy of Medicine, 1930.

The technology to facilitate medical education, like healthcare itself, encounters constant change. Since the beginning, the Delaware Academy of Medicine has quickly adopted new tools to keep pace with healthcare and deliver vital new information to professionals and the public. The Delaware Academy of Medicine Historical Archives preserves a collection from a oncerevolutionary technology that is now declared obsolete: Tel-Med.

Events like the 1962 Health Fair had 65,000 visitors, and other well-attended health education projects such as the Health Forums demonstrated a need to share medical and dental with the public. ${ }^{1}$ Governor Pierre S. du Pont made the first call to Tel-Med in a small press conference on March 31, 1980. The Delaware Academy of Medicine established the State's Tel-Med consumer health program franchise to give the public free and anonymous access to health-related information entirely by telephone. The Tel-Med program was even more popular than the Academy initially planned, receiving nearly 30,000 calls in the first six weeks of operation. By the end of the first year, Tel-Med received over 143,000 calls with an average of 12,000 calls per month (statistically 1 out of every 4 Delawareans), making it one of the most heavily used TelMed programs in the country. ${ }^{2}$

When Tel-Med first opened the phone lines, the caller had access to a library of 204 pre-recorded audio tapes on a wide variety of medical and dental topics. Dr. Joyce Brothers and other personalities lent their voices to the appropriate subjects. By 1996 there were 898 topics from which to choose (see figure 1). To select a topic, the caller referred to a number-coded directory printed in the blue pages of the telephone book. A tape librarian would answer the call, enter the topic code into the Teletronix Information System to connect the caller to the requested tape. The Teletronix System integrated telephone and tape playback equipment into a single console allowing a librarian to operate up to fifteen phone lines. According to Teletronix, the system provided a 75:1 ratio of volume, meaning that it would take 75 people to disseminate the same amount of information that one tape librarian can handle. ${ }^{3}$

Figure 1: Tel-Med Topic Directory Flyer 


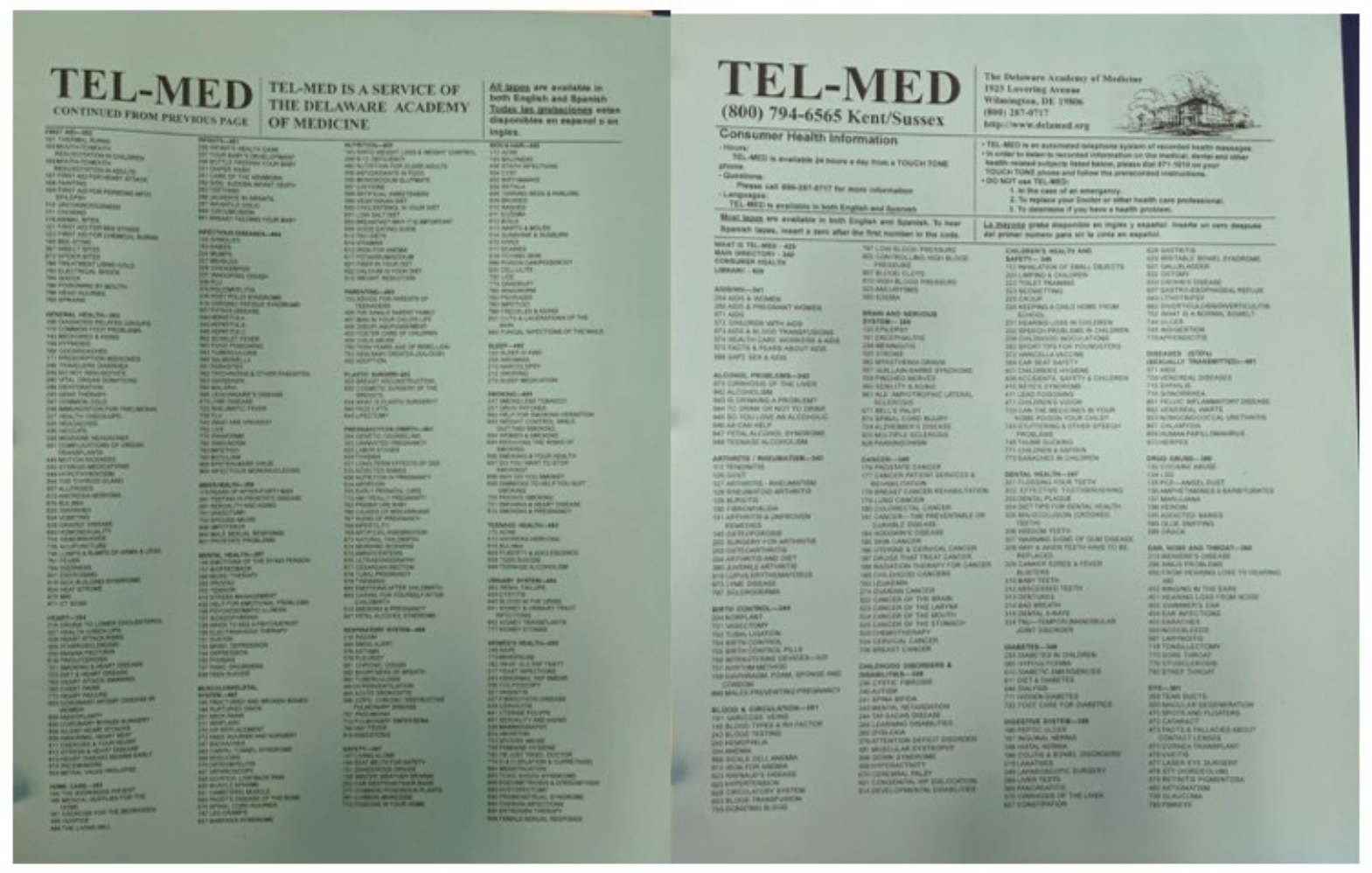

This high-tech program was not cheap. A private local foundation gave a grant to the Academy to cover the initial costs for equipment and installation. The Medical-Dental Bureau of New Castle County provided space for the Tel-Med equipment and made available operators staff it around the clock, seven days a week. There was no charge for this service except during heavy calling volume when additional operators were needed. The Academy appealed to both medical and non-medical organizations in the area for assistance with ongoing operating costs. The Medical Society of Delaware, the New Castle County Medical Society, and ten other organizations responded by "sponsoring" 71 more tapes in addition to the 133 unsponsored tapes. In 1983, a second phone number was added to the program to handle Kent and Sussex counties (figure 2).

Figure 2: Governor Pierre S. duPont ready to make the first phone call, Tel-Med Kick-off for Kent General, June 21, 1983. 


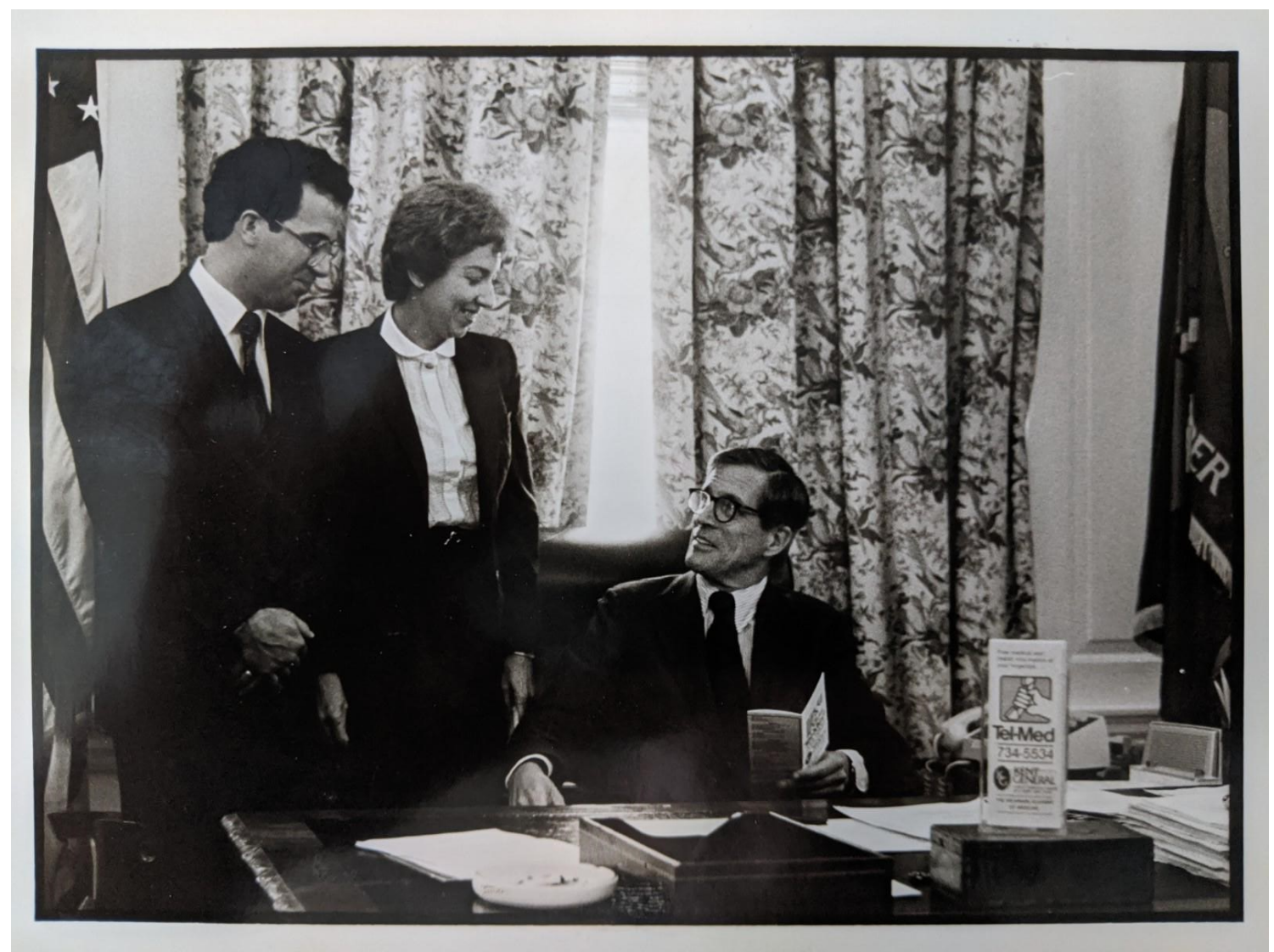

One of the most appealing features of Tel-Med was its anonymity. Callers had access to information that would answer sensitive questions they might otherwise be too embarrassed to ask. In the first three quarters of 1992 the top five tape requests were: 1) "Am I Really Pregnant?" (requested 2,134 times); 2) "AIDS" (2,054 times); 3) "Signs of a Problem Pregnancy" (1,384 times); 4) "Homosexuality" (1,245 times); and 5) "Facts and Fears about AIDS" (1,181 times) (figure 3). The information in each pre-recorded three to five-minute-long tape was written by physicians or other specialists on that topic. Tel-Med provided each subscribing organization with transcripts for review.

Figure 3: Tel-Med call summary from internal Academy report, 1992. 


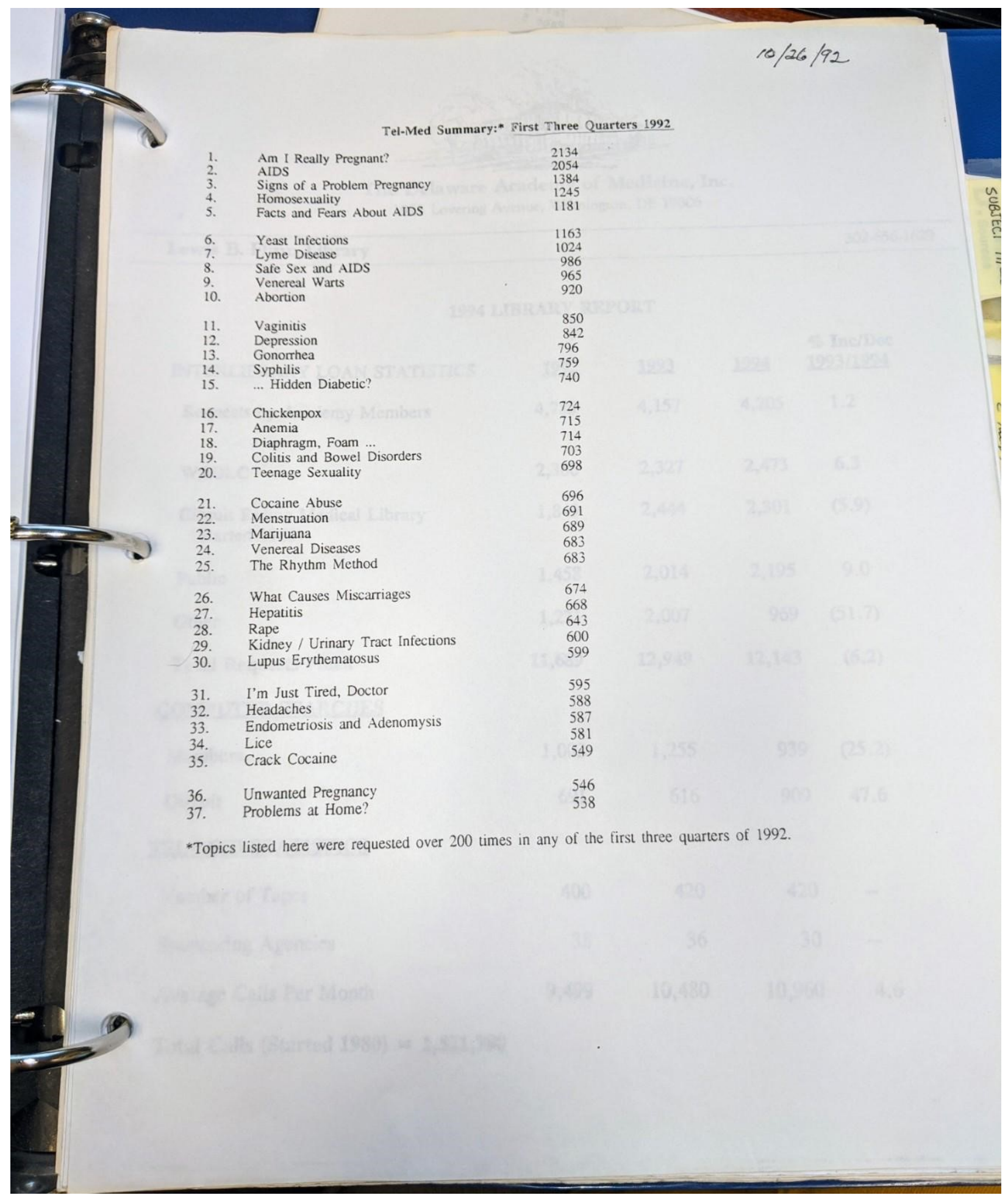

Within ten years, the Teletronix system was outdated. In 1991, the Tel-Med program was computerized entirely to handle the nearly 6,000 monthly calls. The Tel-Med Information System (TMIS) was a state-of-the-art system that stored pre-recorded information and delivered messages to a touch-tone caller. By the late 1990s, users could also go to the World Wide Web to retrieve health information from delamed.org or email consumer@delamed.org. Despite the increasing popularity of the internet in the mid-2000s and the availability of reliable health 
information websites like WebMD.com, the Tel-Med system continued to be one of the Academy's most widely-used consumer programs. Ultimately, the internet took over, rendering the Tel-Med system obsolete, and the Academy discontinued the service.

\section{References}

1. Flinn, Dr. R. (1978, Oct 17). Request for Grant to Crystal Foundation [Letter to Dr. Burt Pratt].

2. Dill, W. G., \& Gill, G. P. (1981, September). Tel-Med in Delaware: A progress report. [Delaware Academy of Medicine Historical Archives.]. Delaware Medical Journal, 53(9), 465-466. PubMed

3. Teletronix Information Systems. (1978, January 24). Answer to Information Request [Letter to Eugene Syrovatka]

\section{Copyright (c) 2021 Delaware Academy of Medicine / Delaware Public Health Association.}

This is an Open Access article distributed under the terms of the Creative Commons Attribution Non-Commercial License (https://creativecommons.org/licenses/by-nc-nd/4.0/) which permits unrestricted non-commercial use, distribution, and reproduction in any medium, provided the original work is properly cited. 